\title{
Fractional Excretion of Phosphate
}

National Cancer Institute

\section{Source}

National Cancer Institute. Fractional Excretion of Phosphate. NCI Thesaurus. Code

C114221.

The determination of the fractional excretion of hosphate that is computed based upon the concentrations of hosphate and creatinine in both blood and urine. 